\title{
Non-invasive prostate cancer detection by measuring expression level of miR-21 and miR-214 in urine
}

\section{Alireza Emamvirdizadeh}

Islamic Azad University Tehran Medical Sciences

Faranak Jamshidian

Islamic Azad University Tehran Medical Sciences

Mehrdad Hashemi

Islamic Azad University Tehran Medical Sciences

\section{Saghi Nooraei}

Islamic Azad University Tehran Medical Sciences

Maliheh Entezari ( $\nabla$ mentezari@iautmu.ac.ir)

Islamic Azad University Tehran Medical Sciences

https://orcid.org/0000-0003-4191-3351

\section{Research article}

Keywords: Prostate cancer, Urine, Biomarker, miR-21, miR-214

Posted Date: July 24th, 2020

DOI: https://doi.org/10.21203/rs.3.rs-44812/v1

License: (c) (1) This work is licensed under a Creative Commons Attribution 4.0 International License. Read Full License

Version of Record: A version of this preprint was published at International Journal of Cancer Management on April 21st, 2021. See the published version at https://doi.org/10.5812/ijcm.110014. 


\section{Abstract}

Background: Prostate cancer is the most prevalent cancer among men worldwide. Diagnosis in this cancer is primarily done using aggressive methods, such as biopsy. Laboratory methods, such as measurement of prostate specific antigen (PSA) in the blood, do not have high sensitivity and specificity. MicroRNAs, a group of diagnostic biomarkers, can be used to diagnose diseases such as cancer. MicroRNA is small, non-coding, single-stranded RNA with a length of 21-23 nucleotides. The present study was undertaken to investigate changes in the expression level of miR-21 and miR-214 in the urine to detect prostate cancer.

Methods: Testing was done on 70 urine samples from prostate cancer patients (32 metastatic and 38 non-metastatic) and 30 from healthy individuals with negative biopsy reports as the control group. Changes in the expression level of miR-21 and miR-214 in the urine were investigated by using qRT-PCR.

Results: miR-21 showed a significant increase in expression $(p=0.003)$ and miR-214 showed a significant decrease in expression $(p=0.000)$ over the results of the control group. The specificity, sensitivity and AUC for combined panels of both microRNAs were $100 \%, 72.14 \%$ and 0.721 and for PSA were $63.33 \%, 61.43 \%$ and 0.620 , respectively.

Conclusions: The results show that miR-21 and miR-214 show significant changes in expression in patients with prostate cancer compared to the control group. A combined panel of miR-21 and miR-214 can be used as a non-invasive method for detecting prostate cancer with higher sensitivity and specificity than the PSA test.

\section{Background}

Prostate cancer is the most common cancer in men and usually occurs in men over 50 years of age (1). Men have a $10 \%$ risk of developing this cancer, of which about $3 \%$ of cases are fatal (2). The primary symptoms are frequent urination, intermittent and poor urine flow, urinary incontinence, blood in the urine, semen excretion with pain, persistent pain in the lower back and impotence (3). Digital rectal examination (DRE) and biopsy are more common than laboratory tests for measuring prostate specific antigen (PSA) in the blood.

PSA is a glycoprotein produced both in cancerous and healthy epithelial cells that helps break down collagen in the semen and make it clot. In the case of prostate disease, over $4 \mathrm{ng} / \mathrm{ml}$ (recognized as normal) leaks into the bloodstream. PSA testing for diagnosis of prostate cancer has shown low sensitivity and specificity. For prostatitis and benign prostatic hyperplasia (BPH), the level of PSA may increase but, even with prostate cancer, it may be normal $(1,4)$.

A DRE shows changes in the shape and size of the prostate, but is not sensitive (5). If the results of PSA and DRE are not normal, prostate cancer is generally confirmed by biopsy. It is the gold standard for detection of prostate cancer, but is highly invasive, costly and painful $(6,7)$. One of the most effective 
factors in increasing patient longevity to improve treatment and reduce the financial and mental costs to the patient is the quick and early diagnosis of cancer. Researchers continue to search for such a method and have identified numerous biomarkers for the detection and tracking of cancer. One of the most important is microRNA.

MicroRNA is small, non-coding single-stranded RNA with a length of 21-23 nucleotides that is involved in the regulation of post-transcriptional expression of about $60 \%$ of human genes. They also regulate processes such as the proliferation, apoptosis, evolution and differentiation of cancers and increase and decrease gene expression. This means that they can be used as biomarkers in the prognosis and diagnosis of diseases, including cancer, in the blood, urine and tissue (8). They are found in plants, animals, and viruses, but they do not exist in bacteria (9). MicroRNA can directly regulate oncogenes and tumor osteoporosis can play an oncogenic or tumor-assisted role, depending on the type of mRNA function they perform (10).

Early evidence of the association between microRNA and cancer was presented by Calin in 2002. $\mathrm{He}$ showed that, in many patients with chronic lymphocytic leukemia, a piece of the genome had been removed, including the miR-15a and miR-16 gene cluster (11). The profile of the expression of the microRNA involved in prostate cancer was published in 2007 (12).

One type of microRNA that plays a role in prostate cancer is miR-21. This microRNA can inhibit expression of tumors by PTEN gene suppressor and increases the aggressive proliferation of cancer cells (13). This microRNA is classified as an incomer. The most important target mRNAs are PDCD4, TPM1, TIMP3, MARCK and PTEN $(14,15)$. It plays an important role in bone metastasis in the advanced stages of prostate cancer (16) and is located on chromosome 17 (17). The miR-214 plays a role in prostate cancer, and the most important target mRNAs are EZH2 and CTNNB1 (14). It is located on chromosome 1 (17).

The aim of the present study was to determine changes in the expression of miR-21 and miR-214 in urine samples of patients with prostate cancer (metastatic and non-metastatic) compared to healthy control subjects, to carry out ROC curve analysis for each microRNA and compare them with the results of the PSA test, to investigate a non-invasive method for diagnosing prostate cancer.

\section{Methods}

\subsection{Collection of urine samples}

Urine samples were collected from suspected prostate cancer patients who referred to Hashemi-Nejad Hospital in Tehran, Iran for biopsies from April 2017 to March 2018. The urine samples were transferred to the Molecular Genetics Lab of the Cancer Institute of Imam Khomeini Hospital (Tehran, Iran) and stored at $4^{\circ} \mathrm{C}$ for up to 3 hours and then were centrifuged at $3500 \mathrm{rpm}$ for $5 \mathrm{~min}$ at $4^{\circ} \mathrm{C}$. Urine samples were stored at $-80^{\circ} \mathrm{C}$ until completed. Based on the pathology report, 70 urine samples were diagnosed 
with prostate cancer (32 metastatic and 38 non-metastatic). 30 urine samples were from healthy participants who had received negative biopsy results.

\subsection{Exclusion and inclusion criteria}

Inclusion criteria: Patients diagnosed with prostate cancer (according to pathology reports) who have no other related diseases. All participants have been informed and completed a consent form and a questionnaire before undergoing a biopsy. This study has been approved by the ethics committee of Islamic Azad Tehran Medical Sciences University-Pharmacy and Pharmaceutical Branches Faculty.

Exclusion criteria: Patients who were sampled after a biopsy, patients received any treatment such as chemotherapy and radiotherapy and patients with related diseases such as bladder and kidney diseases were excluded.

\subsection{RNA extraction from urine}

RNA was extracted by using $500 \mu \mathrm{L}$ of Trizol (Invitrogen; USA) in $2 \mathrm{~mL}$ of urine samples according to manufacturer protocol. A NanoDrop 2000 spectrophotometer (Thermo Scientific; USA) was used to detect concentration and purity of extracted RNA $(\mathrm{ng} / \mu \mathrm{L})$ and RNA with OD260/OD280 ratio between 1.8-2 was taken. The extracted RNA was stored at $-80^{\circ} \mathrm{C}$ until cDNA synthesis.

\section{4 cDNA synthesis}

RNA was converted to cDNA by using a Pars Genome MiR-Amp kit (Iran) according to manufacturer protocol. The CDNA was stored at $-20^{\circ} \mathrm{C}$ until qRT-PCR was performed.

\subsection{Quantitative reverse transcription PCR (qRT-PCR)}

The expression level of miR-21 and miR-214 were measured for 70 prostate cancer patients (32 metastatic and 38 non-metastatic) and 30 healthy participants using Sybr Green Master mix (Takara; Japan) by using Real-time PCR system (Bioneer; South Korea). Urine samples were normalized to internal standard control U6snRNA. This method was chosen for its precision, high sensitivity and low cost. The forward and reverse primer sequences for miR-21, miR-214 and internal reference (U6SnRNA) were designed (Table1).

The reaction was carried out in a volume of $14 \mu \mathrm{L}$ which included $7 \mu \mathrm{L}$ of Sybr Green Master mix, $1 \mu \mathrm{L}$ primer (10 pmol), $3 \mu \mathrm{L}$ cDNA diluted 1 to 4 and $3 \mu \mathrm{L}$ of distilled water.

The Real-time PCR conditions was as follows: Polymerase activation at $95^{\circ} \mathrm{C}$ for $12 \mathrm{~min}$, denaturation at $95^{\circ} \mathrm{C}$ for $15 \mathrm{sec}$, annealing $60^{\circ} \mathrm{C}$ for $30 \mathrm{sec}$ and extension at $72^{\circ} \mathrm{C}$ for $15 \mathrm{sec}$ for $42 \mathrm{cycles}$. The experiment was repeated three times. For each microRNA and U6snRNA, no template control (NTC) was provided which lacked a template (CDNA) for reproduction. PCR performance was calculated using the standard curve and serial dilutions of $107 \%$. Finally, $2 \%$ agarose gel electrophoresis was used to ensure the reproduction and specificity of the components. 
Table 1. Primer Sequences of miR-21, miR-214 and U6SnRNA.

\begin{tabular}{|c|c|c|}
\hline MicroRNA & Forward & Reverse \\
\hline miR-21 & 5'-GTGCAGGGTCCGAGGT-3' & 5'-GCCGCTAGCTTATCAGACTGATGT-3' $^{\prime}$ \\
\hline miR-214 & 5'-GCGGCACAGCAGGCACAGACA-3' & 5'-TGCAGGG \\
\hline U6SnRNA & 5'-CTCGCTTCGGCAGCACA-3' & 5'-AACGCTTCACGAATTTGCGT -3' $^{\prime}$ \\
\hline
\end{tabular}

\subsection{Statistical analysis}

The $2^{-\Delta \Delta C t}$ method was used for pre-processing and fold-change calculations. Analysis of data from Real-time PCR was performed by using REST 2009 software. All statistical calculations and charts were performed using SPSS 22 and $p<0.05$ was considered significant. The sensitivity and specificity of each of the miRNAs was calculated by using Medcalc18.2.1 software and the receiver operating characteristic (ROC) curve was plotted and the AUC calculated. All the box plots represent miRNA levels relative to U6snRNA and they are plotted by using formula $2^{-\Delta C t}$ in Medcalc18.2.1

\section{Results}

The clinical and pathological data of the patients with prostate cancer and healthy controls with negative biopsy results are based on the pathology results shown (Table 2). The presence of miR-21 and miR-214 was identified in the prostate cancer (PCa) samples and miR-21 and miR-214 showed significant change in the expression of PCa patients compared to the control group.

Table 2. Clinical and pathological characteristics of patients with Pca and controls. 


\begin{tabular}{|c|c|c|c|c|}
\hline Group & $\begin{array}{c}\text { Patients with } \\
\text { Pca }\end{array}$ & $\begin{array}{c}\text { Metastatic } \\
\text { group }\end{array}$ & $\begin{array}{c}\text { Non-metastatic } \\
\text { group }\end{array}$ & Controls \\
\hline Number & 70 & 32 & 38 & 30 \\
\hline $\begin{array}{c}\text { [ean (years) } \pm \text { SEM } \\
\text { Range }\end{array}$ & $\begin{array}{c}69.83 \pm 1.03 \\
45-87\end{array}$ & \begin{tabular}{|c|}
$70.62 \pm 1.68$ \\
$45-87$
\end{tabular} & $\begin{array}{c}69.16 \pm 1.29 \\
48-86\end{array}$ & \begin{tabular}{|c|}
$65.97 \pm 1.32$ \\
$52-84$
\end{tabular} \\
\hline $\begin{array}{l}\text { PSA Mean }(\mathrm{ng} / \mathrm{ml}) \pm \\
\text { SEM } \\
\text { Range }\end{array}$ & $\begin{array}{c}18.03 \pm 3.77 \\
1-170.6\end{array}$ & $\begin{array}{c}31.39 \pm 7.64 \\
4.7-170.6\end{array}$ & $\begin{array}{c}6.77 \pm 0.51 \\
1-21\end{array}$ & \begin{tabular}{|c|}
$7.08 \pm 0.53$ \\
$2-13.2$
\end{tabular} \\
\hline $\begin{array}{c}\text { ason score Mode } \\
\text { Range }\end{array}$ & $\begin{array}{c}7 \\
6-10\end{array}$ & $\begin{array}{c}8 \\
7-10\end{array}$ & $\begin{array}{c}7 \\
6-7\end{array}$ & - \\
\hline $\begin{array}{c}\text { Grade Mode } \\
\text { Range }\end{array}$ & $\begin{array}{c}2 \\
1-5\end{array}$ & $\begin{array}{c}4 \\
3-5\end{array}$ & $\begin{array}{c}2 \\
1-2\end{array}$ & - \\
\hline
\end{tabular}

\subsection{Expression of miR-21 in the urine samples of people with prostate cancer}

The miR-21 increased significantly $(p=0.003)$ in the prostate cancer group compared with the control group with a fold change of 3.493. The expression of miR-21 in the metastatic and non-metastatic prostate cancer subgroups was examined and was significantly different. The metastatic subgroup showed a significant increase in expression $(p=0.042)$ compared to the non-metastatic subgroup $(p=$ 0.036). The rate of expression (fold change) miR-21 in the metastatic subgroup was 3.911 and in the nonmetastatic subgroup was 3.176 (Fig. 1).

\subsection{Expression of miR-214 in the urine samples of people with prostate cancer}

The miR-214 showed a significant decrease in expression $(p=0.000)$ in patients with prostate cancer with a fold change of 0.008 . Expression of miR-214 in the metastatic and non-metastatic prostate cancer subgroups was investigated. In the metastatic subgroup, the expression level (fold change) was 0.007 and, in the non-metastatic subgroup, the expression level (fold change) was 0.011 . The metastatic subgroup showed a significant decrease in expression $(p=0.000)$ compared to non-metastatic subgroup $(p=0.000)$ (Fig. 2).

The expression of miR-214 and miR-21 in the prostate cancer and control groups was calculated separately by using the formula $2^{-\Delta \mathrm{Ct}}$ and is plotted in Fig. 3 .

\subsection{The ROC curve analysis of miR-21, miR-214 and PSA}


The ROC curve was used to determine the specificity and sensitivity of miRNA for identification of prostate cancer. This statistical analysis shows the efficacy of miRNA as diagnostic biomarker. The specificity index provides the ability to diagnose a cancer sample from among non-cancerous samples. The sensitivity index confirms its ability to diagnose the disease for the patient. The area under the curve (AUC) indicates the biomarker's prediction power.

In ROC curve analysis, miR-21 showed $100 \%$ specificity, $64.29 \%$ sensitivity and AUC $=0.643$ and miR-214 showed $100 \%$ specificity, $84.29 \%$ sensitivity and AUC $=0.843$. The combined panels of miR-21 and miR214 had $100 \%$ specificity, $72.14 \%$ sensitivity and AUC $=0.721$. PSA showed $63.33 \%$ specificity, $61.43 \%$ sensitivity and AUC $=0.620$ and compared to miRNA, it show less specificity and sensitivity (Fig. 4 and Table 3).

Table 3. Information of ROC Curve analysis.

\begin{tabular}{|c|c|c|c|c|}
\hline & MiR-21 & MiR-214 & $\begin{array}{c}\text { MiR-21 and } \\
\text { MiR-214 }\end{array}$ & PSA \\
\hline Specificity & $100 \%$ & $100 \%$ & $100 \%$ & $63.33 \%$ \\
\hline Sensitivity & $64.29 \%$ & $84.29 \%$ & $72.14 \%$ & $61.43 \%$ \\
\hline AUC & 0.643 & 0.843 & 0.721 & 0.620 \\
\hline P-Value & 0.0133 & 0.0001 & 0.0001 & 0.0464 \\
\hline 95\% CI & $0.541-0.736$ & $0.756-0.908$ & $0.654-0.782$ & $0.517-0.715$ \\
\hline Standard Error & 0.0577 & 0.0438 & 0.0380 & 0.0600 \\
\hline
\end{tabular}

\section{Discussion}

Changes in lifestyle and eating habits, alcohol and tobacco use, electromagnetic radiation, environmental pollution have increased the incidence of cancer year by year. Most diagnostic methods are invasive or do not have high specificity and sensitivity.

In recent years, a large number of diagnostic biomarkers in the biological samples of patients have been introduced for the diagnosis of cancer. These biomarkers are often proteins or nucleic acid, but they have low specificity and sensitivity, are unstable and expensive. Problems with laboratory review mean that most biomarkers are seldom used or approved by the World Health Organization. miRNA is a new generation of diagnostic biomarkers, including in tumor tissue, show different expression patterns for cancer. The pattern of expression of miRNA in human cancers is tissue-dependent and miRNA is significantly more stable under severe environmental factors such as salt, alkali, proteases and nucleases $(18,19)$. Because microRNA circulating in the blood and other biological fluids are more stable with a longer half-lives than proteins, their profile can be used to differentiate cancer from other diseases (20). Biological fluids such as blood and urine are convenient and non-invasive vehicles for collection and 
provide good samples for laboratory and diagnostic research. Finding miRNA biomarkers in such fluids that can accurately detect cancer is a major goal of researchers.

Stuopelyte et al. in Lithuania studied the expression of miR-21, miR-19a and miR-19b in tissue and urine specimens. The expression of miRNA was evaluated by RT-qPCR. The results of this study showed that the miRNAs were expressed in the tissue and in the urine sedimentation of the prostate cancer group. Urinary miR-21 has a significant potential for differentiation of prostate cancer patients and individuals with $\mathrm{BPH}$. The combined panels of the three urine miRNA types has more power to detect prostate cancer compared to the PSA (21).

Foj et al. in Spain studied the expression of miR-21, miR-141, miR-214, miR-375 and let-7c on 60 urine specimens of patients with prostate cancer and 10 healthy subjects. Their results showed that miR-21 ( $p$ $=0.001$ and $A U C=0.817)$, miR-141 $(p=0.033$ and $A U C=0.712)$ and miR-375 ( $p=0.038$ and $A U C=$ $0.707)$ increased and miR-214 ( $p=0.049$ and AUC $=0.716)$ showed low expression levels. No significant difference was found for let-7c $(p=0.185$ and AUC $=0.636)$. Combined panels of miR-21 and miR-375 have been proposed for differentiation of cancerous and healthy patients $(p<0.0001$ and AUC $=0.872)$ (22).

Koppers-Lalic et al. in the Netherlands studied the expression levels of miR-21, miR-204 and miR-375 on 48 urine specimens for prostate cancer patients and 26 urine samples from healthy subjects performed by qRT-PCR method. The results showed that all three miRNA types increased in patients with prostate cancer. Ultimately, the miR-21, miR-204 and miR-375 miR-375 combination had a sensitivity of $72.9 \%$, $88 \%$ specificity and AUC $=0.866$ compared to the PSA test having a sensitivity of $70.8 \%, 60 \%$ specificity and $A U C=0.707(23)$.

The results of the present research showed that miR-21 in patients with prostate cancer increased significantly $(p=0.003)$ in comparison with the healthy subjects. It was shown that the expression of this miRNA was significantly different between the metastatic and non-metastatic subgroups. In the metastatic subgroup $(p=0.036)$, the expression was higher than in the non-metastatic subgroup $(p=$ 0.042). The miR-214 showed a significant decrease in expression in the prostate cancer group $(p=0.000)$ compared with the healthy control group. The results between the metastatic and non-metastatic subgroups indicated that expression of miR-214 was significantly different between groups and was lower in the metastatic subgroup $(p=0.000)$ than in the non-metastatic subgroup $(p=0.000)$.

The results of ROC curve analysis showed that miR-214 had greater sensitivity and diagnostic power than miR-21 and the combination panel of the two miRNA types had greater diagnostic ability and higher specificity and sensitivity than the PSA test. Compared to other methods, such as biopsy, it is superior. The results of this study were consistent with the results of other studies.

\section{Conclusion}


The miR-21 and miR-214 combined panels can be used as a non-invasive diagnostic method for diagnosis of prostate cancer. It is hoped that, with further research on miRNA and cancer, the use of miRNA as a non-invasive and accurate diagnostic method will replace current diagnostic tests in laboratories. Early diagnosis of cancer before metastasis can allow earlier treatment and increase the life expectancy of the affected patients.

\section{List Of Abbreviations}

PSA: Prostate Specific Antigen

miR: MicroRNA

PCR: Polymerase Chain Reaction

DRE: Digital Rectal Examination

ROC: Receiver Operating Characteristic

PCa: Prostate Cancer

AUC: Area Under The Curve

\section{Declarations}

\section{Ethics approval:}

The study was approved by the ethics committee of Islamic Azad Tehran Medical Sciences UniversityPharmacy and Pharmaceutical Branches Faculty with Ethics approval number: IR.IAU.PS.REC.1397.112.

\section{Consent to participate:}

All participants have been informed and completed a consent form and a questionnaire and also approved by the Ethics Committee.

\section{Consent for publication:}

Not Applicable

\section{Availability of data and material:}

The datasets used and/or analysed during the current study are available from the corresponding author.

\section{Competing interests:}

The authors declare that they have no conflict of interest. 


\section{Funding:}

No fund

\section{Authors' contributions:}

AR E: Founder of Project and Manuscript writing

F J: Data analysis

M H: Data collection

S N: Samples Collection

M E: Supervisor of Project

All authors have read and approved the manuscript.

\section{Acknowledgments:}

The authors would like to express their gratitude to the Cancer Institute of Imam Khomeini Hospital (Tehran, Iran) and the Department of Radiology at Hashemi-Nejad Hospital (Tehran, Iran).

\section{References}

1. Huang Y, Li Z-Z, Huang Y-L, Song H-J, Wang Y-J. Value of free/total prostate-specific antigen (F/T PSA) ratios for prostate cancer detection in patients with total serum prostate-specific antigen between 4 and $10 \mathrm{ng} / \mathrm{mL}$ : A meta-analysis. Medicine. 2018;97(13).

2. Turnpenny P, Ellard S. Emerys Elements Of Medical Genetics. Fifteenth ed: Elsevier;2017.

3. Carter H. partin AW. Diagnosis and staging of prostate cancer. Campbell-Walsh Urology 8th ed Sydney: Elsevier Health Sciences. 2002.

4. Guo T, Wang X, Fu H, Tang Y, Meng B, Chen C. Early diagnostic role of PSA combined miR-155 detection in prostate cancer. European review for medical and pharmacological sciences. 2018;22:1615-21.

5. Steggall MJ. Digital rectal examination. Nursing Standard (through 2013). 2008;22(47):46.

6. Kachakova D, Mitkova A, Popov E, Popov I, Vlahova A, Dikov T, et al. Combinations of serum prostatespecific antigen and plasma expression levels of let-7c, miR-30c, miR-141, and miR-375 as potential better diagnostic biomarkers for prostate cancer. DNA and cell biology. 2015;34(3):189-200

7. Arriaga-Canon C, De La Rosa-Velázquez IA, González-Barrios R, Montiel-Manríquez R, Oliva-Rico D, Jiménez-Trejo $F$, et al. The use of long non-coding RNAs as prognostic biomarkers and therapeutic targets in prostate cancer. 2018. 
8. Song CJ, Chen H, Chen LZ, Ru GM, Guo JJ, Ding QN. The potential of microRNAs as human prostate cancer biomarkers: A meta-analysis of related studies. Journal of cellular biochemistry. 2018;119(3):2763-86

9. Wang H, Peng R, Wang J, Qin Z, Xue L. Circulating microRNAs as potential cancer biomarkers: the advantage and disadvantage. Clinical epigenetics. 2018;10(1):59.

10. Croce $\mathrm{CM}$. Causes and consequences of microRNA dysregulation in cancer. Nature reviews genetics. 2009;10(10):704

11. Calin GA, Dumitru CD, Shimizu M, Bichi R, Zupo S, Noch E, et al. Frequent deletions and downregulation of micro-RNA genes miR15 and miR16 at 13q14 in chronic lymphocytic leukemia. Proceedings of the National Academy of Sciences. 2002;99(24):15524-9.

12. Porkka KP, Pfeiffer MJ, Waltering KK, Vessella RL, Tammela TL, Visakorpi T. MicroRNA expression profiling in prostate cancer. Cancer research. 2007;67(13):6130-5.

13. Yang Y, Guo J-X, Shao Z-Q. miR-21 targets and inhibits tumor suppressor gene PTEN to promote prostate cancer cell proliferation and invasion: An experimental study. Asian Pacific journal of tropical medicine. 2017;10(1):87-91.

14. Sandiford OA, Moore CA, Du J, Boulad M, Gergues M, Eltouky H, et al. Human Aging and Cancer: Role of miRNA in Tumor Microenvironment. Exosomes, Stem Cells and MicroRNA: Springer; 2018. p. 13752

15. Pang Y, Young CY, Yuan H. MicroRNAs and prostate cancer. Acta Biochim Biophys Sin. 2010;42(6):363-9.

16. Massillo C, Dalton GN, Farré PL, De Luca P, De Siervi A. Implications of microRNA dysregulation in the development of prostate cancer. Reproduction. 2017;154(4):R81-R97.

17. ncbi.nlm.nih.gov [Internet].

18. Calin GA, Croce CM. MicroRNA signatures in human cancers. Nature reviews cancer. 2006;6(11):857.

19. Xi Y, Nakajima G, Gavin E, Morris CG, Kudo K, Hayashi K, et al. Systematic analysis of microRNA expression of RNA extracted from fresh frozen and formalin-fixed paraffin-embedded samples. Rna. 2007;13(10):1668-74.

20. D'Souza AL, Chevillet JR, Ghanouni P, Yan X, Tewari M, Gambhir SS. Tumor characterization by ultrasound-release of multiple protein and microRNA biomarkers, preclinical and clinical evidence. PloS one. 2018;13(3):e0194268.

21. Stuopelytè K, Daniūnaite K, Jankevičius F, Jarmalaitè S. Detection of miRNAs in urine of prostate cancer patients. Medicina. 2016;52(2):116-24.

22. Foj L, Ferrer F, Serra M, Arévalo A, Gavagnach M, Giménez N, et al. Exosomal and Non-Exosomal Urinary miRNAs in Prostate Cancer Detection and Prognosis. The Prostate. 2017;77(6):573-83.

23. Koppers-Lalic D, Hackenberg M, De Menezes R, Misovic B, Wachalska M, Geldof A, et al. Non-invasive prostate cancer detection by measuring miRNA variants (isomiRs) in urine extracellular vesicles. Oncotarget. 2016;7(16):22566. 
Figures

miR-21 Expression

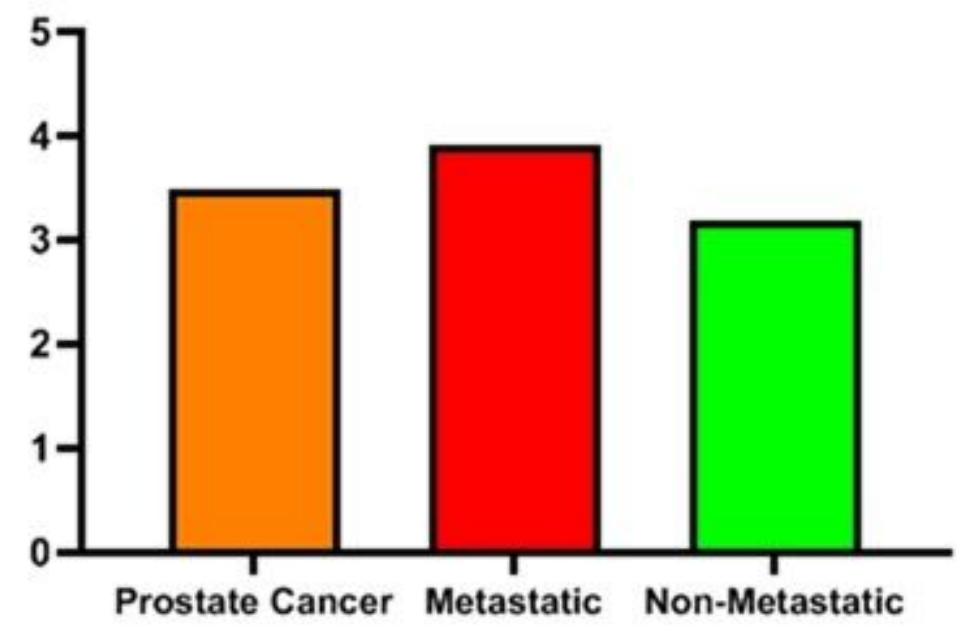

Figure 1

Expression of miR-21 in Prostate cancer, metastatic and Non-metastatic groups.

miR-214 Expression

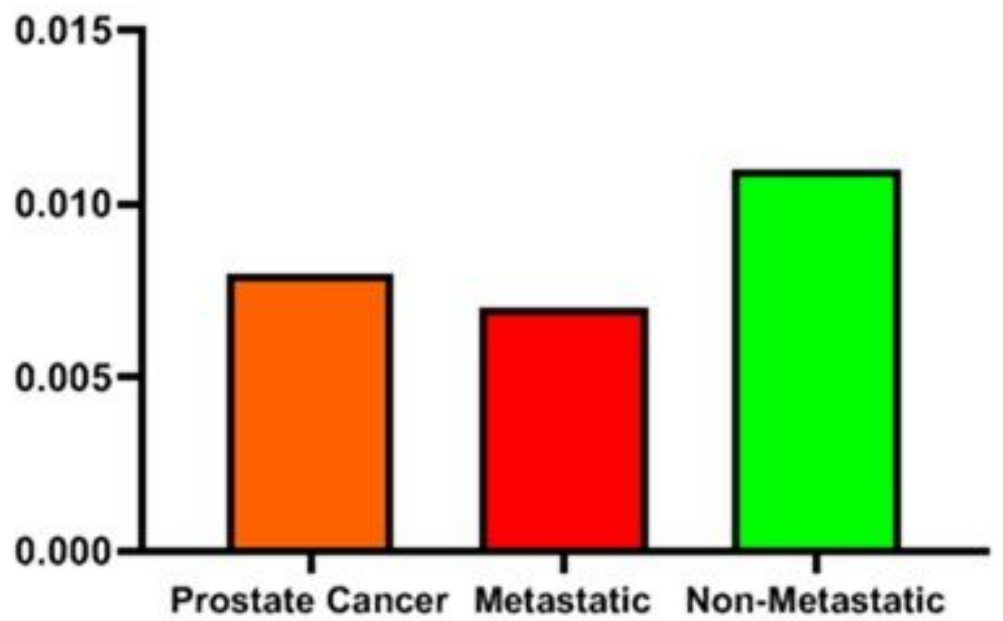

Figure 2

Expression of miR-214 in Prostate cancer, metastatic and Non-metastatic groups. 
A

miR-21

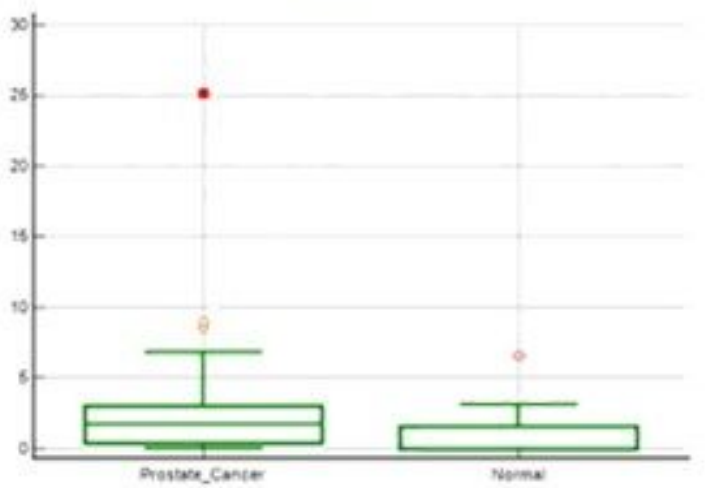

B

miR-214

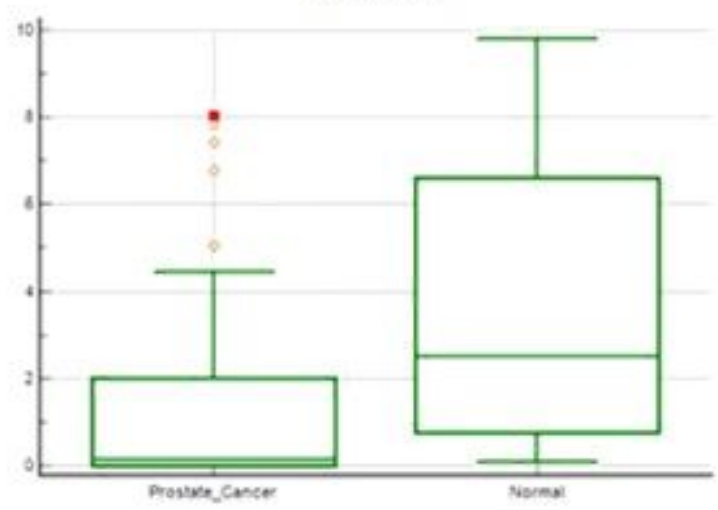

Figure 3

Expression of miR-21 and miR-214 in urine samples: (A) Box plot of 70 urine specimens from patients with prostate cancer that shows increased expression of miR-21 over specimens of 30 from healthy control subjects; (B) miR-214 in the prostate cancer group shows decreased expression in comparison with that of the control group. 
A

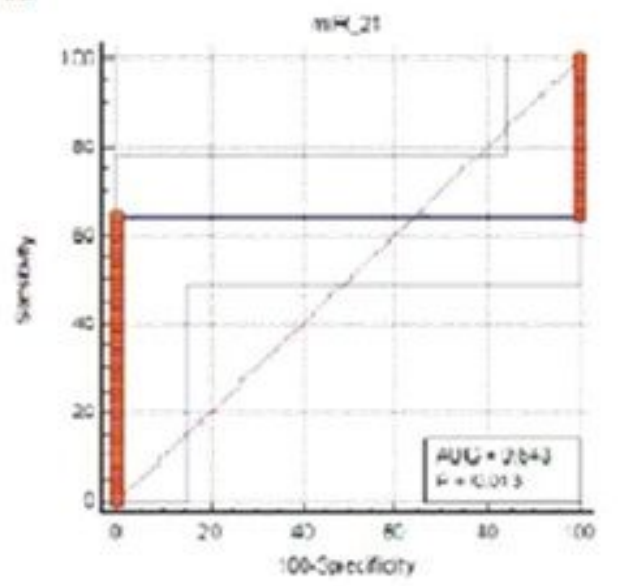

B

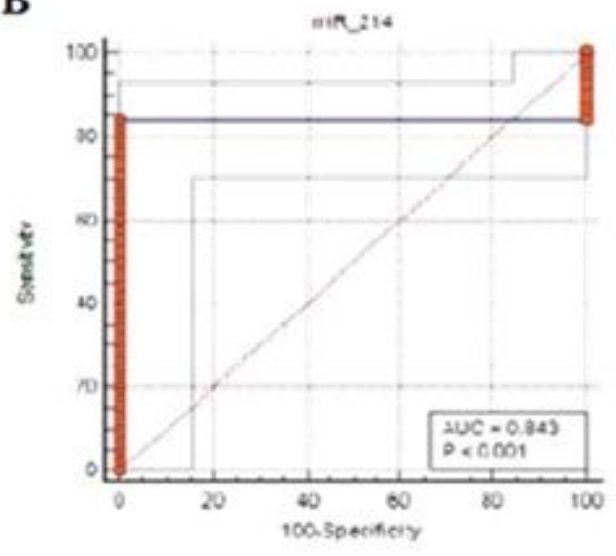

C

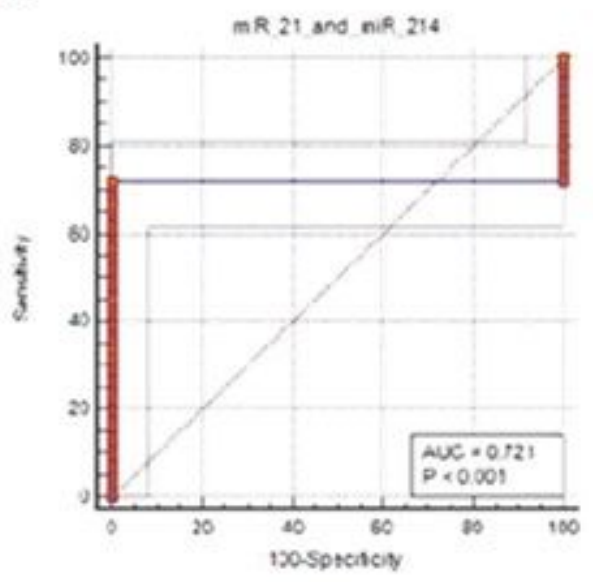

D

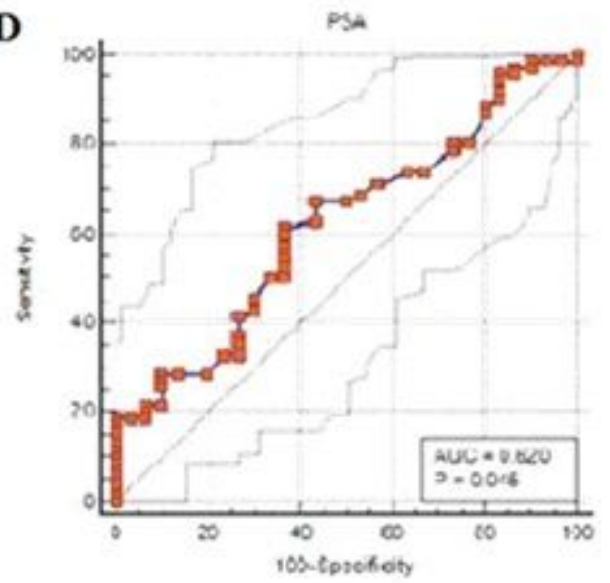

Figure 4

ROC curve analysis. (A) The ROC Curve analysis for miR-21 (B) The ROC Curve analysis for miR-214 (C) The ROC Curve analysis for miR-21+miR-214 (D) The ROC Curve analysis for PSA. 